\title{
A Regular Indirect Boundary Element Method for Thermal Analysis
}

Link to publication record in Manchester Research Explorer

\section{Citation for published version (APA):}

Wearing, J. L., \& Sheikh, M. (1988). A Regular Indirect Boundary Element Method for Thermal Analysis. International Journal for Numerical Methods in Engineering, 25(0), 495-515.

\section{Published in:}

International Journal for Numerical Methods in Engineering

\section{Citing this paper}

Please note that where the full-text provided on Manchester Research Explorer is the Author Accepted Manuscript or Proof version this may differ from the final Published version. If citing, it is advised that you check and use the publisher's definitive version.

\section{General rights}

Copyright and moral rights for the publications made accessible in the Research Explorer are retained by the authors and/or other copyright owners and it is a condition of accessing publications that users recognise and abide by the legal requirements associated with these rights.

\section{Takedown policy}

If you believe that this document breaches copyright please refer to the University of Manchester's Takedown Procedures [http://man.ac.uk/04Y6Bo] or contact uml.scholarlycommunications@manchester.ac.uk providing relevant details, so we can investigate your claim.

\section{OPEN ACCESS}




\title{
A REGULAR INDIRECT BOUNDARY ELEMENT METHOD FOR THERMAL ANALYSIS
}

\author{
J. L. WEARING AND M. A. SHEIKH \\ Department of Mechanical Engineering, University of Sheffield, Sheffield U.K.
}

\begin{abstract}
SUMMARY
A Regular Indirect Boundary Element formulation is developed following a weighted residual approach. In this formulation, the fictitious source density, which appears in the integral equations of the method, is distributed on a surface which is exteriorly separated from the physical field boundary of the problem. This approach does not require the evaluation of singular integrals and produces undeteriorated solutions at geometric discontinuities. The formulation is presented here as applied to two dimensional and axisymmetric thermal problems. It includes a different subregioning scheme which treats each subregion, of a multidomain problem, separately, thereby eliminating the need for a sophisticated reduction scheme.
\end{abstract}

\section{INTRODUCTION}

The principal advantages of Boundary Domain Techniques over domain methods such as the Finite Element Method are the reduction in the number of degrees of freedom required to achieve a satisfactory discretization and selective computation of the interior solution.

Like domain methods, there can be different types of Boundary Domain Techniques, the most distinguished of which are the 'Direct' and 'Indirect' Boundary Element Methods. The Direct Boundary Element Method (DBEM) is generally represented as being based on Green's identity and until now has been considered to be more versatile and general than the Indirect Boundary Element Method (IBEM). ${ }^{1}$ The IBEM may be represented as a special case of DBEM. In this method, the integral equations are expressed in terms of a unit singular solution (or source) which satisfies the governing differential equation. This singular solution is distributed at a certain density over the boundary of the domain of the given problem. ${ }^{2}$ The density functions themselves have no physical significance, but once they are obtained from a numerical solution of the integral equations, the values of the solution parameters within the domain can be calculated from them through simple integrations.

In the IBEM, the primary field variables (e.g. temperatures) and their derivatives (fluxes) are indirectly linked. Unlike the DBEM, it only requires the original integral equations to provide the interior flux solution. The DBEM requires further differentiation of their kernel functions. Although the IBEM is attractive owing to its simplicity, it has a serious drawback when used in its conventional form.

On a smooth boundary a valid solution of the integral equations by the indirect formulation can be obtained. ${ }^{3}$ However, in situations where the boundary has geometric discontinuities (corners), there is no guarantee that the proposed fictitious source density distribution exists. If it does, it is likely to become infinity at the corners and makes modelling in this region very difficult, leading subsequently to errors. This inherent feature of the IBEM has limited its applications, and 
consequently it has been in the shadow of its direct counterpart (DBEM) in spite of having certain more attractive features.

An alternative approach to the conventional indirect formulation is to relocate the surface of the source distribution at a certain distance outside the physical domain of the problem resulting in a system of Regular Integral Equations. ${ }^{4}$ It has been shown that the Regular Indirect Boundary Element Method (RIBEM) eliminates the consistent singular behaviour of the source density at the corners. ${ }^{5}$ In addition, it also relieves the need to apply the special and careful treatment required for the evaluation of singular integrals in the neighbourhood of the singular point. As such, the regular approach enhances the capability and range of applications of the Indirect Method.

In this paper, the Regular Indirect Boundary Element Method (RIBEM) is presented as applied to two-dimensional and axisymmetric thermal analysis, using subregions. In formulating the subregioning scheme, the fact that the primary field variables and their derivatives in the IBEM are indirectly linked is exploited. This allows the matrix inversion to be performed regionally, making it unnecessary to form the final system matrix, and hence allowing large problems to be solved without the use of a special reduction scheme.

Finally, two steady-state heat conduction problems are analysed using the RIBEM to demonstrate its capabilities. Results are compared with Singular IBEM or finite element solutions.

\section{INDIRECT BEM FORMULATION}

For potential problems the governing (Laplace's) equation which is satisfied by the function, $u$, within the domain $\Omega$ of a given problem is

$$
\nabla^{2} u=0 \quad \text { in } \Omega
$$

The boundary conditions for the problem are (Figure 1):

$$
\begin{array}{lll}
u=\bar{u} & \text { on } S_{1} & \text { (essential condition) } \\
q=\bar{q} & \text { on } S_{2} & \text { (natural condition) }
\end{array}
$$

where $q=\partial u / \partial n$ and the total boundary is $S=S_{1}+S_{2}$.

Equation (1) can be transformed into an integral equation for the Direct Boundary Element Method (DBEM) by following a weighted residual approach. ${ }^{6}$ Introducing a weighting function $w$ which has continuous first derivatives and which satisfies the governing equation (1), the

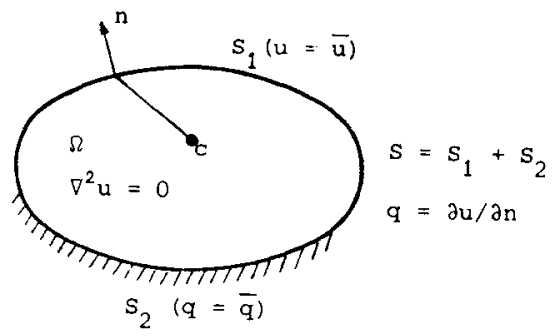

Figure 1. Problem definition-DBEM 
weighted residual statement can be written as

$$
\int_{\Omega}\left(\nabla^{2} u\right) w \mathrm{~d} \Omega=\int_{S_{2}}(q-\bar{q}) w \mathrm{~d} S-\int_{S_{1}}(u-\bar{u}) \frac{\partial w}{\partial n} \mathrm{~d} S
$$

Integrating the Laplacian term of equation (3) by parts twice, gives

$$
\int_{\Omega}\left(\nabla^{2} w\right) u \mathrm{~d} \Omega=-\int_{S_{2}} \bar{q} w \mathrm{~d} S-\int_{S_{1}} q w \mathrm{~d} S+\int_{S_{2}} u \frac{\partial w}{\partial n} \mathrm{~d} S+\int_{S_{1}} \bar{u} \frac{\partial w}{\partial n} \mathrm{~d} S
$$

Assuming a concentrated source at a point $\mathrm{c}$ interior to the domain $\Omega$, the governing equation is

$$
\nabla^{2} w+\Delta^{c}=0
$$

where $\Delta^{\mathrm{c}}$ is the Dirac delta function for which

$$
\int_{\Omega} u\left(\nabla^{2} w+\Delta^{\mathrm{c}}\right) \mathrm{d} \Omega=\int_{\Omega} u\left(\nabla^{2} w\right) \mathrm{d} \Omega+u^{\mathrm{c}}
$$

If equation (5) is to be satisfied by $w$, then

$$
\int_{\Omega} u\left(\nabla^{2} w\right) \mathrm{d} \Omega=-u^{\mathrm{c}}
$$

and equation (4) becomes

$$
u^{\mathrm{c}}+\int_{S_{1}} \bar{u} \frac{\partial w}{\partial n} \mathrm{~d} S+\int_{S_{2}} u \frac{\partial w}{\partial n} \mathrm{~d} S=\int_{S_{1}} q w \mathrm{~d} S+\int_{S_{2}} \bar{q} w \mathrm{~d} S
$$

If the values of $\bar{u}$ and $\bar{q}$ are known for respective parts of the boundary, equation (8) can be written, in general, for the total boundary as

$$
u^{\mathrm{c}}=\int_{s}(q w-u f) \mathrm{d} S
$$

where $f=\partial w / \partial n$. The weighting function $w$ can be taken as the fundamental solution to the governing Laplace's equation which, for an isotropic case in two dimensions, is given as

$$
w=-\frac{1}{2 \pi} \ln r
$$

where ' $r$ ' is the distance between the source point $\mathrm{c}$ and the point under consideration (field point).

Now consider the region $\hat{\Omega}$ (Figure 2) bounded by $S$ but exterior to $\Omega$ and assume $\hat{u}$ to be the

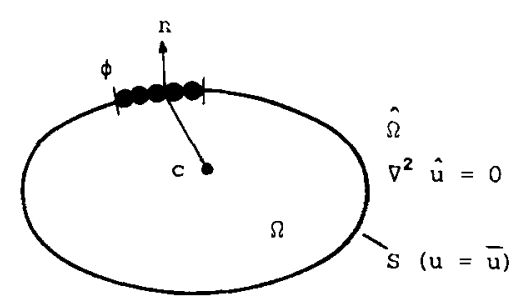

Figure 2. Problem definition-IBEM 
solution of the Laplace's equation

$$
\nabla^{2} \hat{u}=0 \quad \text { in } \hat{\Omega}
$$

Again, following a weighted residual approach and carrying out the integrations over the boundary $S$ and the domain $\hat{\Omega}$ but maintaining the field point at c yields the following equations:

$$
0=\int_{S}(\hat{q} w+\hat{u} f) \mathrm{d} S
$$

which in comparison to equation (9), has

(a) the left hand side term as zero since $\mathrm{c}$ is exterior to $\hat{\Omega}$, and

(b) a reversed sign for the term involving $f$ since the sense of the outward normal to $\hat{\Omega}$ is opposite to that of $\Omega$.

If $\hat{u}$ is specified to be that solution in $\hat{\Omega}$ which established on $S$ exactly the same boundary solution as in the initial interior region problem, then

$$
u=\hat{u} \quad \text { on } S
$$

Substituting equation (13) into equation (12) and adding the result to equation (9) produces

$$
u^{\mathfrak{c}}=\int_{S}(q+\hat{q}) w \mathrm{~d} S
$$

By defining $\phi=q+\hat{q}$, equation (14) becomes

$$
u^{\mathrm{c}}=\int_{S} \phi w \mathrm{~d} S
$$

Thus the 'Indirect' formulation is established from the Direct BEM where $\phi$ represents the initially unknown density of the unit singular solution $(w)$ over $S$. Subsequently,

$$
q^{c}=\int_{S} \phi f \mathrm{~d} S
$$

For steady-state heat conduction problems, the field quantities $u$ and $q$ are temperature and flux respectively. In order to formulate the problem as a boundary technique, the point $c$ needs to be taken on the boundary. By bringing this field point onto the surface, equation (15) provides the temperature field which is continuous everywhere. Similariy, equation (16) is satisfied everywhere except when the source point and the field point coincide. However, equation (16) may be expressed as a Cauchy principal-value integral, and on a smooth boundary

$$
q^{\mathrm{c}}=-\frac{1}{2} \phi^{\mathrm{c}}+\int_{S} \phi f \mathrm{~d} S
$$

where $\phi^{\mathrm{c}}$ is the value of the source density at point $\mathrm{c}$.

Equations (15) and (17) forms the basis of numerical modelling in the Conventional (Singular) Indirect Boundary Element Method. However, apart from the requirement of evaluating certain singular integrals, which add to the computer overhead, this approach suffers from one major drawback. In problems where the boundary possesses corners, the source density $\phi$ becomes infinity at some of these locations, leading to serious modelling difficulties. ${ }^{7}$ Accordingly, some earlier workers ${ }^{2}$ have approximated the geometry by representing corners by two independent nodes placed slightly away from the actual corner. This approach has the effect of rounding off the 
corner, thereby approximating the source density to take some finite value. However, it has a disadvantage in that it results in poor solutions at the corners and edges for all problems, irrespective of their field behaviour.

\section{REGULAR INDIRECT BEM}

An alternative to the above 'singular' approach is the Regular Indirect Boundary Element Method (RIBEM), in which the source surface is separated from the field boundary and moved outwardly, as shown in Figure 3. This operation results in the integral equations being regular everywhere.

$$
\begin{aligned}
& \text { Hence } \quad u^{\mathrm{c}}=\int_{S} \phi w \mathrm{~d} S \\
& \text { and } \left.\quad q^{\mathrm{c}}=\int_{S} \phi f \mathrm{~d} S\right\}
\end{aligned}
$$

It has been found that by such treatment the infinite source density behaviour at the corners is eliminated. ${ }^{8}$

\section{Numerical solution}

The regular boundary integral equation (equation (18)) itself is a statement of the exact solution to a given problem. However, for most problems, having irregular boundaries, an analytical solution of equation (18) is not possible and a numerical solution has to be sought. Any errors are due therefore to discretization, numerical approximations and subsequent solution of the algebraic equations. The source density $\phi$ remains the actual unknown of the system's equations.

If $N_{\mathrm{c}}$ collocation or field points are distributed over the field boundary, $S_{\mathrm{c}}$, and the source surface $S_{\mathrm{s}}$ is divided into $n$ segments, as shown in Figure 4, for a collocation point c the integral

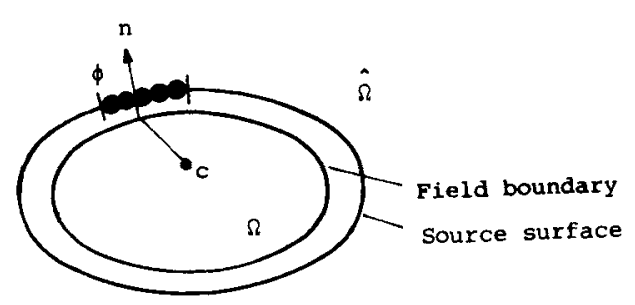

Figure 3. Problem definition-RIBEM

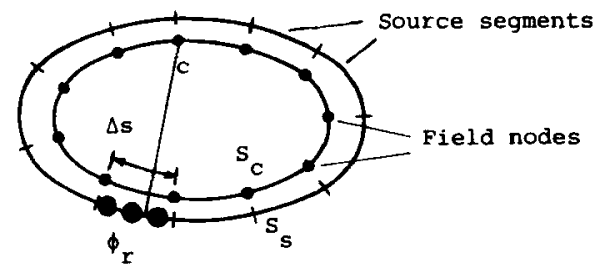

Figure 4. Discretization-RIBEM 
equation (18) can be written in discretized form as

and

$$
\left.\begin{array}{rl}
u^{\mathrm{c}} & =\sum_{r=1}^{n} \int_{\Delta S} w_{\mathrm{cr}} \phi_{r} \mathrm{~d} S \\
q^{\mathrm{c}} & =\sum_{r=1}^{n} \int_{\Delta S} f_{\mathrm{cr}} \phi_{r} \mathrm{~d} S
\end{array}\right\}
$$

where $\Delta S$ is a segment of the physical field boundary

$\phi_{r}$ is the variation of source density over the source segment ' $r$ '

$w_{c r}$ is the fundamental solution relating the collocation point ' $c$ '

and the source segment ' $r$ ', and $f_{c r}$ its derivatives.

If a quadratic variation is defined for the source (Figure 5), then $\phi_{r}$ can be expressed in terms of the nodal values $\phi_{i}$ using the shape functions $N_{r i}$, i.e.

$$
\phi_{r}=N_{r i} \phi_{i}
$$

where

$$
\begin{aligned}
\phi_{i} & =\left[\begin{array}{l}
\phi_{1} \\
\phi_{2} \\
\phi_{3}
\end{array}\right] \text { and } N_{r i}=\left[\begin{array}{lll}
N_{1} & N_{2} & N_{3}
\end{array}\right] \\
N_{1} & =2\left(\xi-\frac{1}{2}\right)(\xi-1) \\
N_{2} & =-4 \xi(\xi-1) \\
N_{3} & =2 \xi\left(\xi-\frac{1}{2}\right)
\end{aligned}
$$

where $\xi$ is the local location of the source point on segment ' $r$ ' of length $L$. Thus equation (19) now becomes

$$
\begin{aligned}
& u^{\mathfrak{c}}=\sum_{r=1}^{n}\left[\int_{\Delta S} w_{\mathrm{cr}} N_{r i} \mathrm{~d} S\right] \phi_{i} \\
& q^{\mathfrak{c}}=\sum_{r=1}^{n}\left[\int_{\Delta S} f_{\mathrm{cr}} N_{r i} \mathrm{~d} S\right] \phi_{i}
\end{aligned}
$$

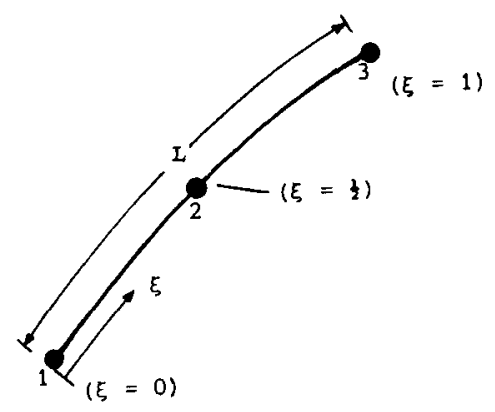

Figure 5. Quadratic element 
These equations can be written as

$$
\text { and } \left.\quad \begin{array}{l}
{[u]=[W][\phi]} \\
{[q]=[F][\phi]}
\end{array}\right\}
$$

In the point collocation method of boundary satisfaction, the number of collocation points must be equal to the number of source nodes. At each node, either the temperature or the flux boundary condition is prescribed, leading to a system of algebraic equations from equation (22).

\section{Location of the source surface}

It is theoretically possible to locate the sources corresponding to the collocation points at any distance outside the domain in the RIBEM. However, in practice this is not quite possible. It is undesirable to have the sources too near the boundary as the singular nature of the fundamental solutions necessitates special treatment of integration in the neighbourhood of the sources. On the other hand, having the sources too far away from the boundary increases the significance of rounding errors and linear dependency. Therefore, a region is sought between the two extremes which yields a stable solution (Figure 6).

In the present algorithm for the RIBEM, only the geometry of the physical boundary of the problem $\left(S_{\mathrm{c}}\right)$ is defined. Each segment of the source surface is then formed from the corresponding segment of $S_{\mathrm{c}}$ by shifting the segment along the outward normals to a distance determined by a chosen non-dimensional factor $\lambda$. The actual distance $R_{r}$ between the two surfaces is given as

$$
R_{r}=\lambda L_{r} / 2
$$

where $L_{r}$ is the length of the local $r$ th element as shown in Figure 7.

It is obvious that in a situation where two adjacent segments on the field boundary are of different length, the corresponding source segments will be discontinuous. Such modelling is possible, as has been shown by the successful employment of discontinuous elements in the Direct Boundary Element Method. ${ }^{9}$ It is adopted here to maintain a constant $R / L$ ratio throughout the length of the boundary. In addition, apart from the corner segments, adjacent segments are conditioned to share the same value of nodal source density at their nearest nodes. This reduces the number of degrees of freedom. An alternative approach is to maintain the same distance between the two surfaces throughout the boundary.

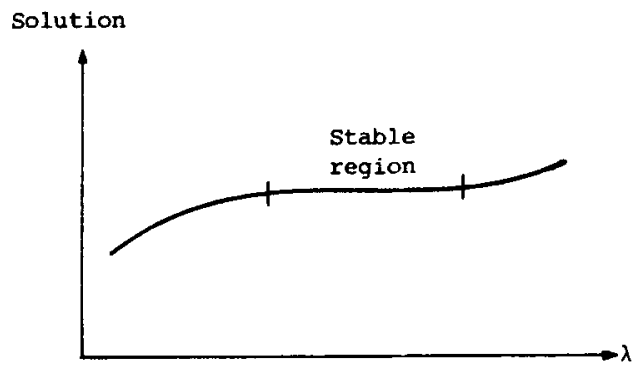

Figure 6. Variation of solution with source surface location (given by $\lambda$ ) in RIBEM 


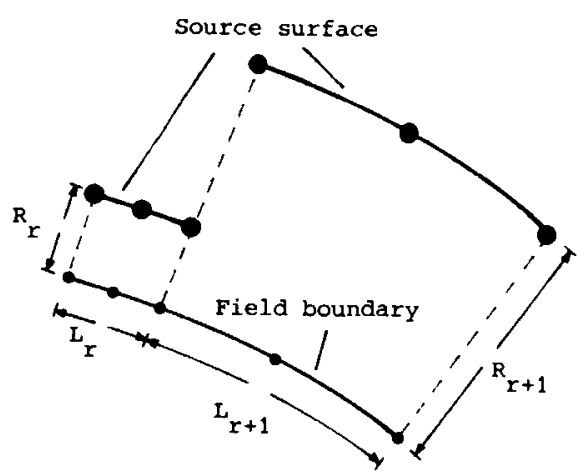

Figure 7. Separation of source surface from physical field boundary in RIBEM

\section{Modelling of corners}

In most engineering applications the boundaries have geometric discontinuities such as corners. But as mentioned before, this has been the major source of difficulties in the Singular Indirect Boundary Element Method as the behaviour of the source density is such that it tends to infinity at some of these corners. To overcome this problem, the following strategy for the modelling of corners is adopted in the RIBEM.

Field nodes. In order to give $\phi$ a finite value at the corner, the multiple-node concept is adopted for all types of boundary conditions. This means that the field point is taken slightly away from the corner, thus representing it by two nodes, as shown in Figure 8.

Source nodes. As the source surface is shifted away from the boundary, the distribution of source density nodes at the corner of this surface is achieved, as shown in Figure 9 . The last nodes of the source segments on each side of the corner subtend an angle $\beta$, where $0 \leqslant \beta \leqslant \alpha / 2$. Although the choice of $\beta$ is fairly arbitrary, in the present algorithm it is kept at $\beta=\alpha / 3$.

\section{Axisymmetric problems}

Axisymmetric problems may be represented by a cylindrical co-ordinate system $(r, z)$. The algorithm then becomes essentially a two-dimensional one. ${ }^{2}$

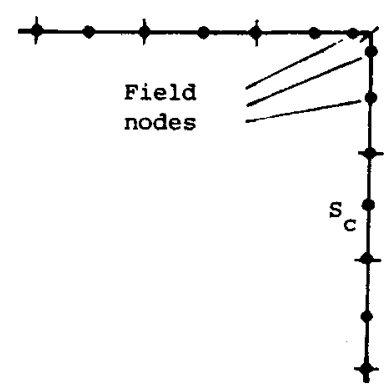

Figure 8. Modelling of physical field boundary around a corner in RIBEM 


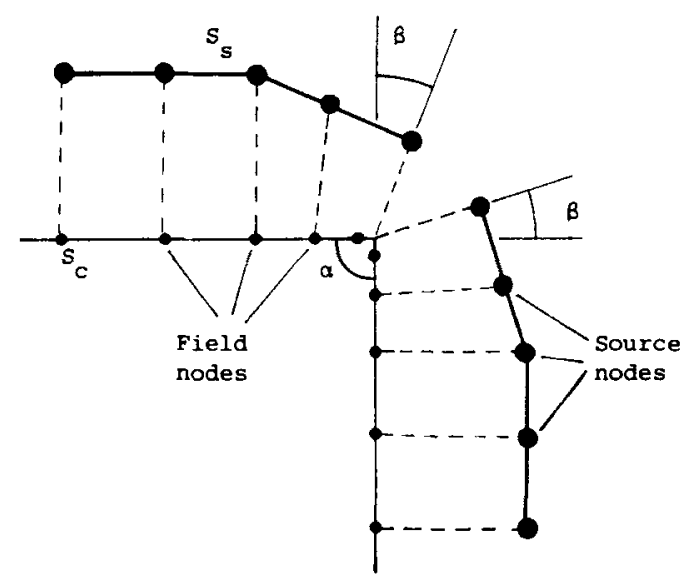

Figure 9. Modelling of source surface around a corner in RIBEM

Using the integral equation in terms of the source $w$ distributed at a specific density $\phi$ over a defined surface, the temperature $u^{\mathrm{c}}(r, z)$ at point $\mathrm{c}$ for axisymmetric problems may be written as

$$
u^{\mathfrak{c}}=\int_{S} r_{0} w \phi \mathrm{d} S
$$

The fundamental solution for a three-dimensional problem is given by

$$
w=\frac{1}{4 \pi r}
$$

The effect due to a ring source acting at radius $r_{0}$ and depth $z_{0}$ (Figure 10) can be obtained by expressing equation (25) in cylindrical co-ordinates $(r, \theta, z)$ and integrating the results with respect to $\theta$ between the limits 0 and $\pi$, so that

$$
w=\frac{1}{2 \pi} \int_{0}^{\pi} \frac{\mathrm{d} \theta}{\sqrt{ } a-b \cos \theta}
$$

where $a=r^{2}+r_{0}^{2}+\left(z-z_{0}\right)^{2}$ and $b=2 r r_{0}$. Equation (26) can be dealt with as an elliptic integral, i.e.

$$
w=\frac{2}{\pi \sqrt{ } a+b} K(m)
$$

where $K(m)$ is a complete elliptic integral of the first kind of modulus $m$ and complementary modulus $m_{1}=m-1$ :

$$
K(m)=\int_{0}^{\pi} \frac{\mathrm{d} \theta}{\sqrt{1-m \sin ^{2} \theta}}
$$

Choosing a polynomial approximation for $K(m)$, as given by Abramowitz and Stegun, ${ }^{10}$

$$
K(m)=\sum_{j=0}^{n}\left[a_{j} m_{1 j}+b_{j} m_{1 j} \ln \left(\frac{1}{m_{1}}\right)\right]+\varepsilon(m)
$$

For $n=4$, the error term $\varepsilon(m) \leqslant 2 \times 10^{-8} ; a_{j}$ and $b_{j}$ are constants. 


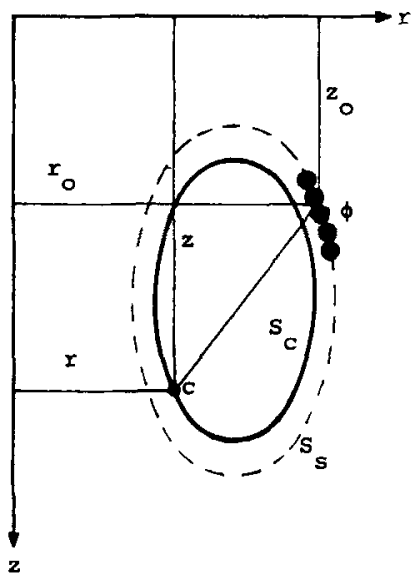

Figure 10. Axisymmetric problems-formulation in RIBEM

The flux at point $\mathrm{c}$ is

$$
q^{\mathrm{c}}=\int_{S} r_{0} f \phi \mathrm{d} S
$$

where

$$
f=-\left[\frac{\partial w}{\partial r} n_{r}+\frac{\partial w}{\partial z} n_{z}\right]
$$

$n_{r}, n_{z}$ are the components of a unit outward normal at point $\mathrm{c}$ in the $r$ and $z$ directions respectively.

By using the identity

$$
E(m)=m_{1}\left[2 m \frac{\mathrm{d} K(m)}{\mathrm{d} m}+K(m)\right]
$$

where $E(m)$ is a complete elliptic integral of the second kind, $f$ can be expressed as

$$
f=n_{r} \frac{[E(m)-K(m)]}{\pi r \sqrt{ } a+b}+\frac{\left[n_{r}\left(r-r_{0}\right)+n_{z}\left(z-z_{0}\right)\right]}{2 \pi(a-b) \sqrt{ } a+b} E(m)
$$

Again, $E(m)$ can be expressed by a polynomial approximation as

$$
E(m)=\sum_{j=1}^{n}\left[c_{j} m_{1 j}+d_{j} m_{1 j} \ln \left(\frac{1}{m_{1}}\right)\right]+\varepsilon(m)
$$

where for $n=4, \varepsilon(m)<2 \times 10^{-8} ; c_{j}$ and $d_{j}$ are constants.

\section{SUBREGIONING}

In the case of problems having large domains and irregular boundaries the use of a single region may have disadvantages. As the system matrix is fully populated it generates a large number of coefficient arrays and the time required to formulate and solve such a system is considerable, and unless an efficient reduction scheme is available there could be a limitation on the problem size. 
Moreover, when analysing long and slender structures the resulting system matrix has a high proportion of insignificant, small off-diagonal terms which implies inefficient computation. The effect of subregioning is to remove the slenderness of the structure (Figure 11). Additionally, when using the Regular Method, problems arise with concave geometries, resulting in a dense accumulation of the source density nodes on the corresponding source surfaces. The use of subregions effectively remove the concave geometries, as shown in Figure 12.

Most conventional subregioning schemes resort to assembling the subregional matrices into one final banded matrix. ${ }^{2}$ However, in the present formulation, a different version of the subregioning scheme is employed which avoids the formulation of a system matrix. Each subregional matrix is inverted and stored immediately after its formulation, thereby eliminating the need to use a reduction scheme. It also means that less computing time is spent on inversions, as the time required to invert $n$ matrices of size $N$ is less than that required to invert a single matrix of size $n N$.

In the Indirect BEM, the field quantities (temperatures and fluxes) are governed by separate integral equations indirectly linked by the distribution of the source density $\phi$. As the interfacial relations of adjacent subregions are between the field quantities and not the source density, this natural feature of the method can be exploited by formulating and inverting the matrices in each subregion separately. The unknowns at a common interface are obtained by solving a set of

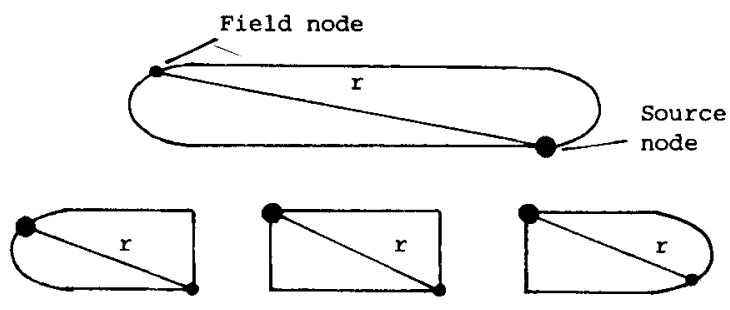

Figure 11. Subregioning-removal of slenderness from structure
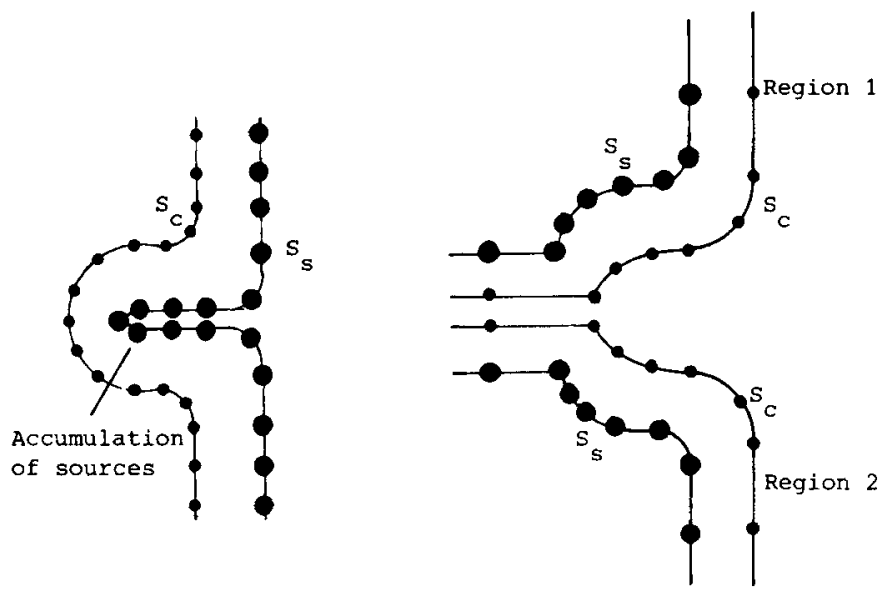

Figure 12. Subregioning in RIBEM--to avoid accumulation of sources in concave sections of the geometry 
simultaneous equations derived from the interfacial relationships

$$
\begin{aligned}
& u_{1}=u_{2} \\
& q_{1}=-q_{2}
\end{aligned}
$$

where subscripts 1 and 2 correspond to regions 1 and 2 respectively. In a problem with two subregions, for example (Figure 13) there will be

$n_{1}$ nodes on the external boundary of region $1\left(S_{11}\right)$,

$n_{2}$ nodes on the external boundary of region $2\left(S_{22}\right)$,

and $n_{12}$ nodes on the interface of regions 1 and $2\left(S_{12}\right)$.

Rewriting equation (22) for region $\Omega_{1}$

$$
\begin{aligned}
& {[u]=\left[W_{1}\right]\left[\phi_{1}\right]} \\
& {[q]=\left[F_{1}\right]\left[\phi_{1}\right]}
\end{aligned}
$$

Applying the boundary conditions,

$$
\left[v_{1}\right]=\left[A_{1}\right]\left[\phi_{1}\right]
$$

where $\left[v_{1}\right]$ consists of prescribed boundary values and interfacial unknowns ( $u$ 's and $q$ 's), and $\left[A_{1}\right]$ consists of corresponding rows of matrices $\left[W_{1}\right]$ and $\left[F_{1}\right]$.

Inverting equation (36),

$$
\left[\phi_{1}\right]=\left[A_{1}^{-1}\right]\left[v_{1}\right]
$$

Substituting equation (37) into equation (35),

$$
\begin{aligned}
& {[u]=\left[W_{1}\right]\left[A_{1}^{-1}\right]\left[v_{1}\right]} \\
& {[q]=\left[F_{1}\right]\left[A^{-1}\right]\left[v_{1}\right]}
\end{aligned}
$$

Separating the known peripheral and the unknown interfacial field variables,

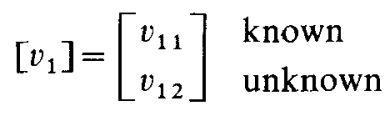

Substituting equation (39) into equation (38) gives

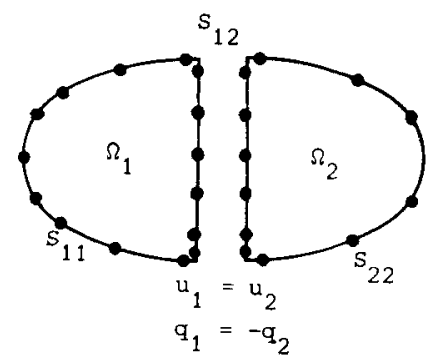

Figure 13. A two subregional problem 


$$
\begin{aligned}
& {[u]=\left[W_{1}\right]\left[A_{11}^{-1}\right]\left[v_{11}\right]+\left[W_{1}\right]\left[A_{12}^{-1}\right]\left[v_{12}\right]} \\
& {[q]=\left[F_{1}\right]\left[A_{11}^{-1}\right]\left[v_{11}\right]+\left[F_{1}\right]\left[A_{12}^{-1}\right]\left[v_{12}\right]}
\end{aligned}
$$

where

$$
\left[A_{1}^{-1}\right]=\left[\begin{array}{ll}
A_{11}^{-1} & A_{12}^{-1}
\end{array}\right]
$$

Similarly, for region $\Omega_{2}$,

$$
\begin{aligned}
& {[u]=\left[W_{2}\right]\left[A_{21}^{-1}\right]\left[v_{21}\right]+\left[W_{2}\right]\left[A_{22}^{-1}\right]\left[v_{22}\right]} \\
& {[q]=\left[F_{2}\right]\left[A_{21}^{-1}\right]\left[v_{21}\right]+\left[F_{2}\right]\left[A_{22}^{-1}\right]\left[v_{22}\right]}
\end{aligned}
$$

where

$$
\left[v_{2}\right]=\left[\begin{array}{l}
v_{21} \\
v_{22}
\end{array}\right] \begin{aligned}
& \text { unknown } \\
& \text { known }
\end{aligned}
$$

and

$$
\left[A_{2}^{-1}\right]=\left[\begin{array}{ll}
A_{21}^{-1} & A_{22}^{-1}
\end{array}\right]
$$

Now, considering the interface $S_{12}$ (or $S_{21}$ ); if it is arbitrarily prescribed by unknown temperatures, then,

and

$$
v_{12} \text { becomes } u_{12}
$$

$u_{21}$ becomes $u_{2}$

and the interfacial relationships at each of the freedom nodes on the interface are given by

$$
\begin{aligned}
& u_{12}=u_{21} \\
& q_{12}=-q_{21}
\end{aligned}
$$

Substituting equations (40b) and (41b) into equation (42b) gives

$$
\left[F_{1}\right]\left[A_{11}^{-1}\right]\left[v_{11}\right]+\left[F_{1}\right]\left[A_{12}^{-1}\right]\left[u_{12}\right]+\left[F_{2}\right]\left[A_{21}^{-1}\right]\left[u_{12}\right]+\left[F_{2}\right]\left[A_{22}^{-1}\right]\left[v_{22}\right]=0
$$

Rearranging equation (43)

$$
\left\{\left[F_{1}\right]\left[A_{12}^{-1}\right]+\left[F_{2}\right]\left[A_{21}^{-1}\right]\right\}\left[u_{12}\right]=-\left\{\left[F_{1}\right]\left[A_{11}^{-1}\right]\left[v_{11}\right]+\left[F_{2}\right]\left[A_{22}^{-1}\right]\left[v_{22}\right]\right\}
$$

Equation (44) can now be used to formulate $n_{12}$ equations to determine $n_{12}$ interfacial temperatures $\left(u_{12}\right)$. Having obtained $u_{12}$, all the coefficients of $\left[v_{1}\right]$ and $\left[v_{2}\right]$ are known. The source densities for each subregion can now be obtained using equation (37). Viewing each of the subregions as a separate problem, the field solution at any point on the boundary or within the subdomains can be computed from its source density distribution using equations (35a) and (35b). The flow chart for this algorithm is presented in Figure 14.

\section{CASE STUDIES}

\section{Heat conduction in a finite fin}

The temperature on the side $\mathrm{AD}$ of the fin (Figure 15) is maintained at $100^{\circ} \mathrm{C}$; the remaining boundary at $0^{\circ} \mathrm{C}$. Although the geometry is simple, the heat flux at points $\mathrm{A}$ and $\mathrm{D}$ is expected to be singular. Conductivity of the medium is set to unity; $\mathrm{AB}=2 \mathrm{~m}, \mathrm{BC}=1 \mathrm{~m}$. Figure 16 shows the mesh employed for the solution of this problem using the Singular and Regular IBEM'S.

Figure 17 shows the variation of flux along $\mathrm{AB}$ for the two methods along with the Fourier series solution. ${ }^{11}$ Table I compares the temperatures in the interior of the domain and flux at the corner node $K$.

It is shown that both methods agree well with the Fourier series solution on the boundary and in the interior of the domain, except at the corners. The error (relative to the Fourier series 


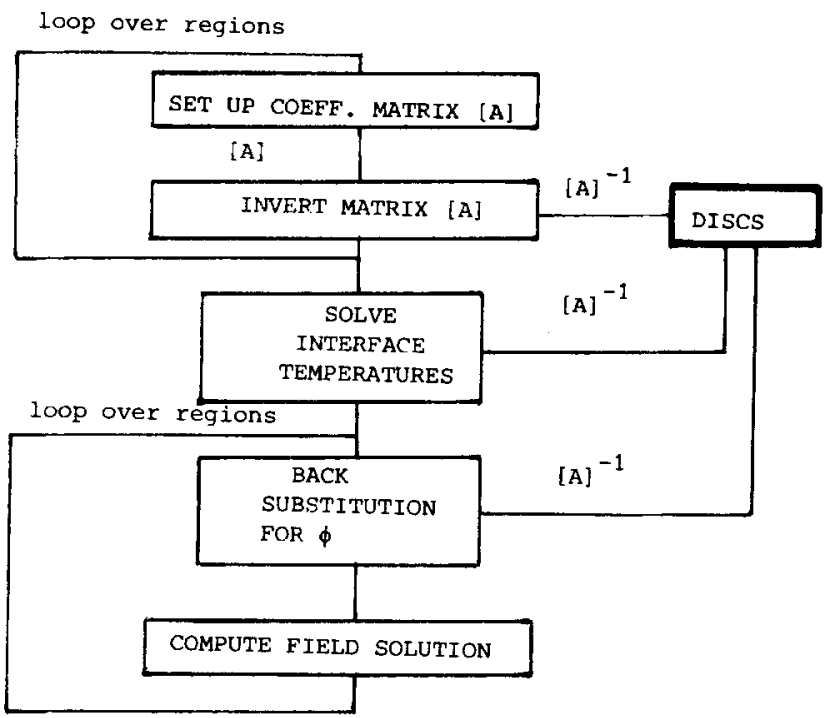

Figure 14. Flow chart for subregioning algorithm in RIBEM

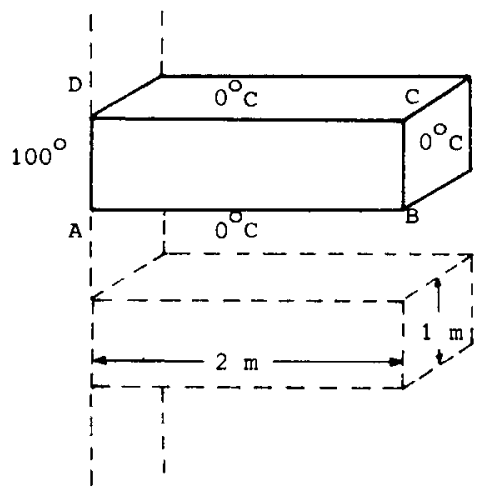

Figure 15. Finite fin-boundary conditions

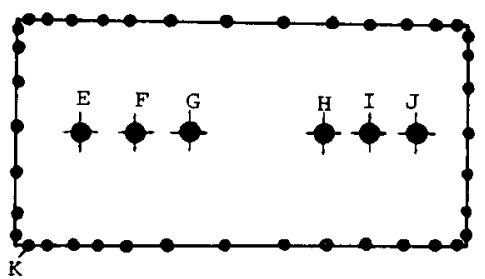

Figure 16. Finite fin--boundary element mesh 


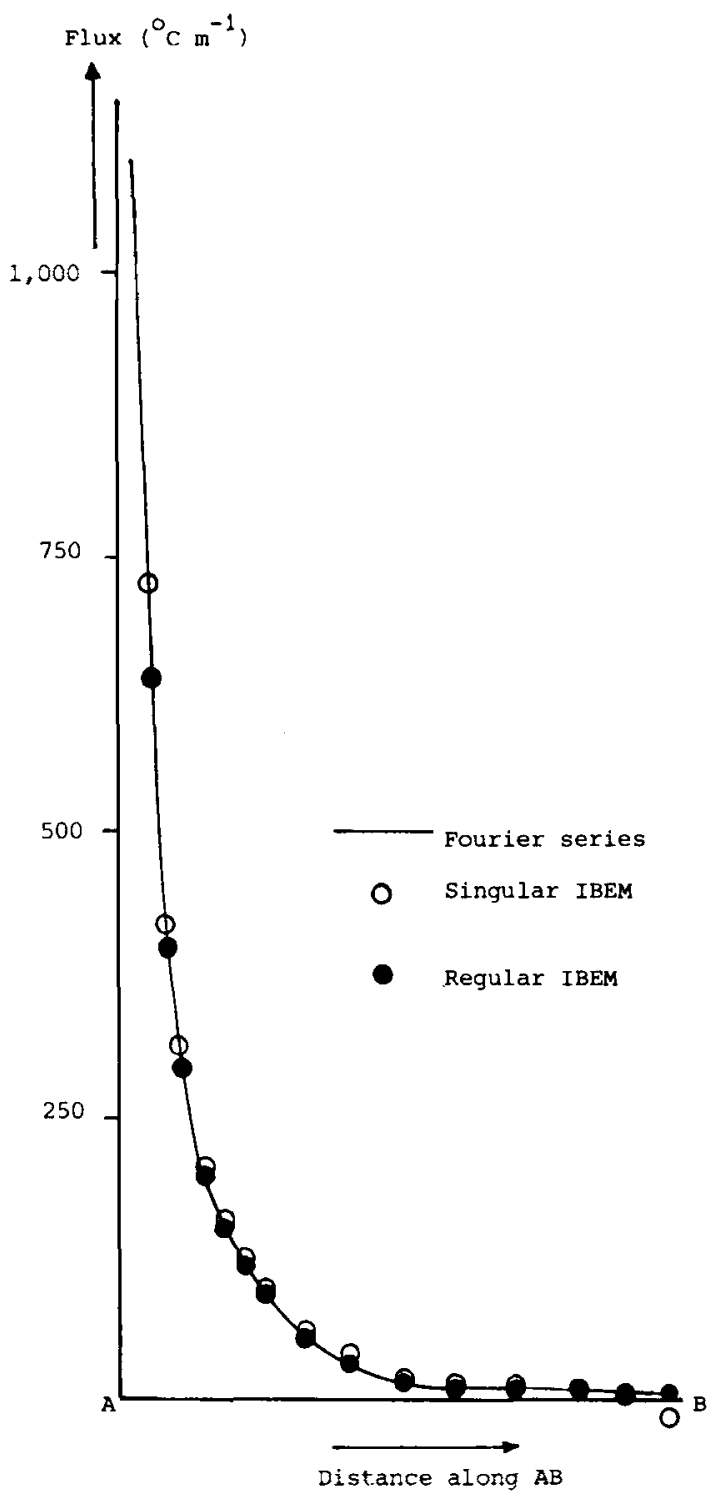

Figure 17. Variation of flux along edge $A B$ of the finite fin

solution) produced in the interior is found to be 0.17 per cent for the Regular Method and 0.64 per cent for the Singular Method. But at the corner node, $K$, the Regular Method produced 2.2 per cent error while the Singular Method gave an error of 17 per cent. Also, the fluxes given by the Singular Method deterioriated at the other corners, B and C. It should be noted that the solution given by the Regular Method was found to be stable for a wide range of source surface locations outside the domain given by $\lambda=0 \cdot 5-6 \cdot 5$. 
Table I. Comparison of solutions for the finite fin problem

\begin{tabular}{llccc}
\hline Point & $\begin{array}{c}\text { Field } \\
\text { variable }\end{array}$ & $\begin{array}{c}\text { Fourier } \\
\text { series } \\
\text { solution }\end{array}$ & $\begin{array}{c}\text { Regular } \\
\text { IBEM } \\
\text { solution }\end{array}$ & $\begin{array}{c}\text { Singular } \\
\text { IBEM } \\
\text { solution }\end{array}$ \\
\hline E & Temp. $\left({ }^{\circ} \mathrm{C}\right)$ & $62 \cdot 400$ & 62.340 & $62 \cdot 290$ \\
$\mathrm{~F}$ & Temp. $\left({ }^{\circ} \mathrm{C}\right)$ & $35 \cdot 300$ & $35 \cdot 250$ & $35 \cdot 220$ \\
$\mathrm{G}$ & Temp. $\left({ }^{\circ} \mathrm{C}\right)$ & $19 \cdot 180$ & $19 \cdot 150$ & $19 \cdot 130$ \\
$\mathrm{H}$ & Temp. $\left({ }^{\circ} \mathrm{C}\right)$ & 1.094 & $1 \cdot 092$ & 1.087 \\
$\mathrm{I}$ & Temp. $\left({ }^{\circ} \mathrm{C}\right)$ & $0 \cdot 518$ & 0.517 & 0.511 \\
$\mathrm{~J}$ & Temp. $\left({ }^{\circ} \mathrm{C}\right)$ & $0 \cdot 152$ & $0 \cdot 152$ & 0.148 \\
\hline $\mathrm{K}$ & Flux $\left({ }^{\circ} \mathrm{C} \mathrm{m}^{-1}\right)$ & $2.947 \times 10^{3}$ & $3.013 \times 10^{3}$ & $3.448 \times 10^{3}$ \\
\hline
\end{tabular}

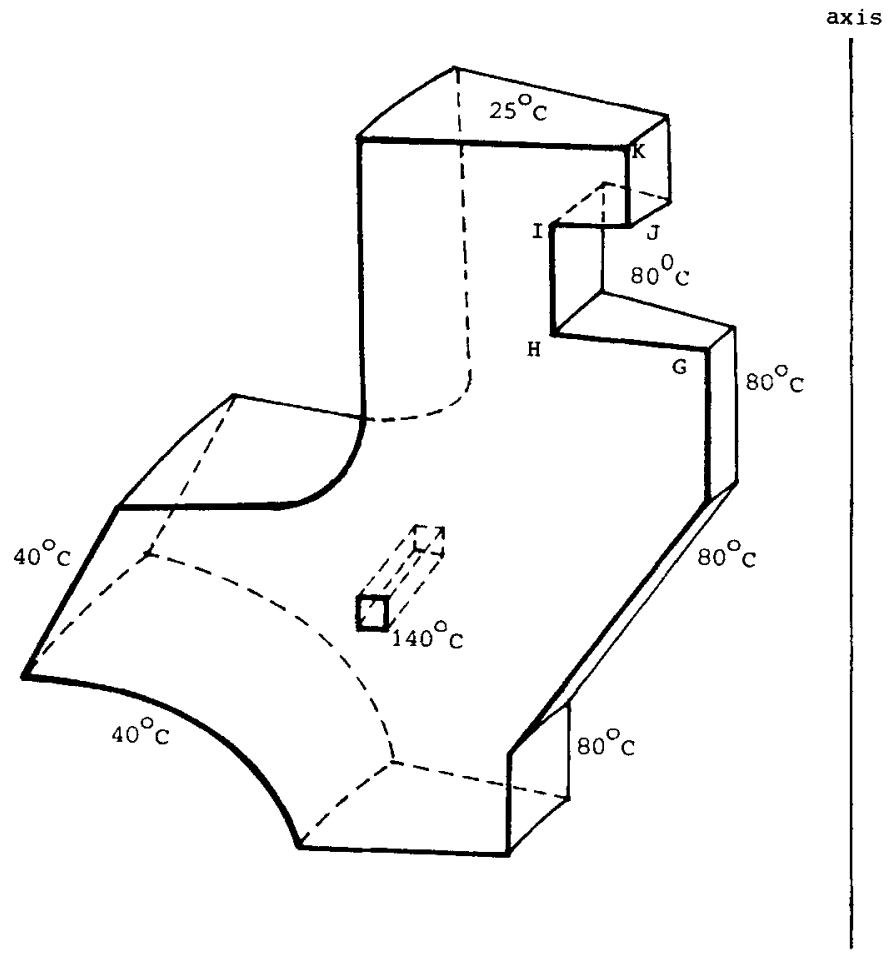

Figure 18. Homogeneous pump casing - boundary conditions

\section{Temperature field in a homogeneous pump casing}

This is an axisymmetric steady state thermal problem for which the boundary conditions are shown in Figure 18. Figure 19 shows the discretized model used for the analysis by the Regular IBEM. It employs five subregions and 105 quadratic elements. Figure 20 shows the mesh employed for the Finite Element Analysis, using 256 quadratic rectangular elements with 865 degrees of freedom. The FEM solution was obtained using the PAFEC 75 package. Figures 21 


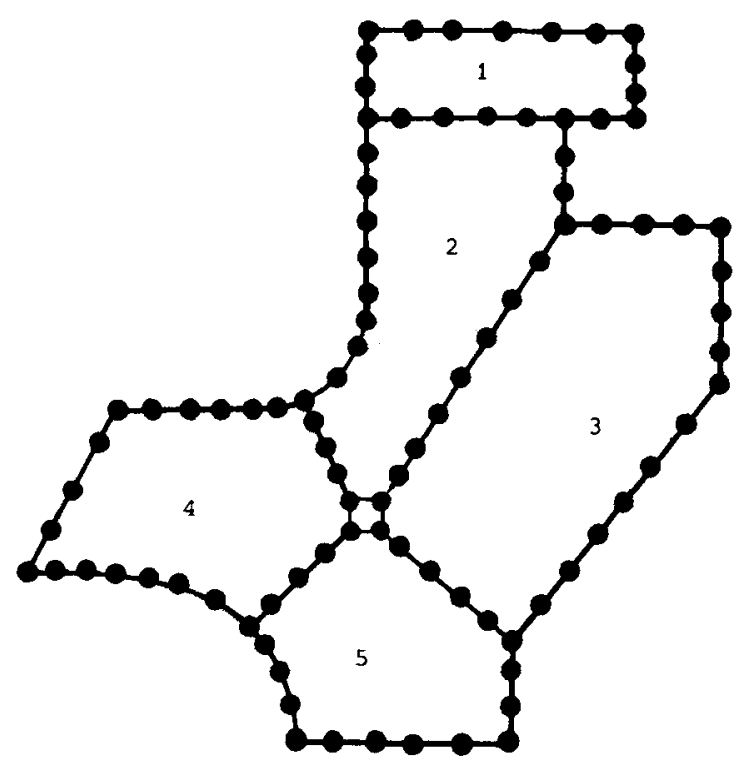

Figure 19. Pump casing-RIBEM model

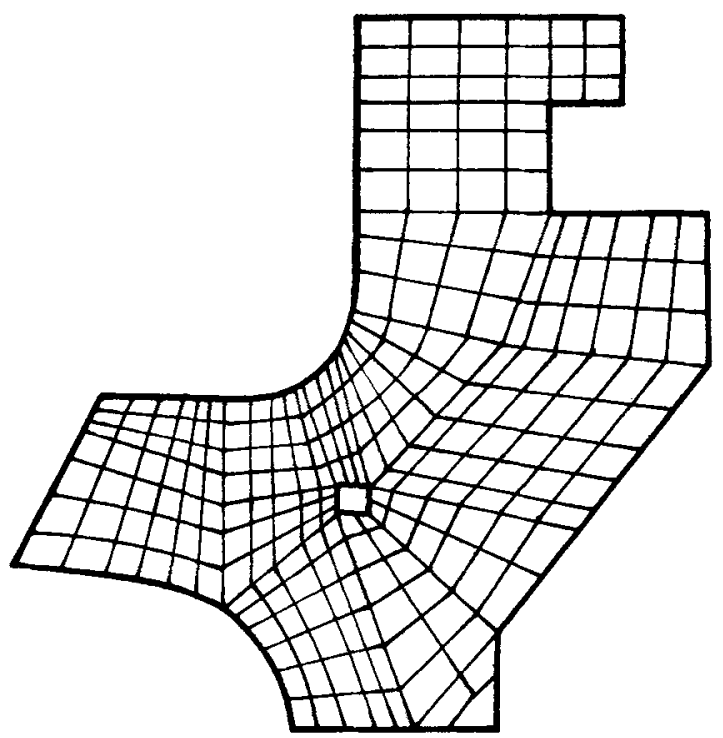

Figure 20. Pump casing-FE model

and 22 show the temperature fields obtained by the RIBEM and FEM Analyses respectively. It is observed that both methods agree well throughout the domain and on the boundary.

Table II compares the computational effort involved in the RIBEM and FEM analyses. Clearly, the Regular Indirect Method was better than the FEM in input data processing and solution times. It is worth noting that considerable effort was involved in arriving at the FE mesh 


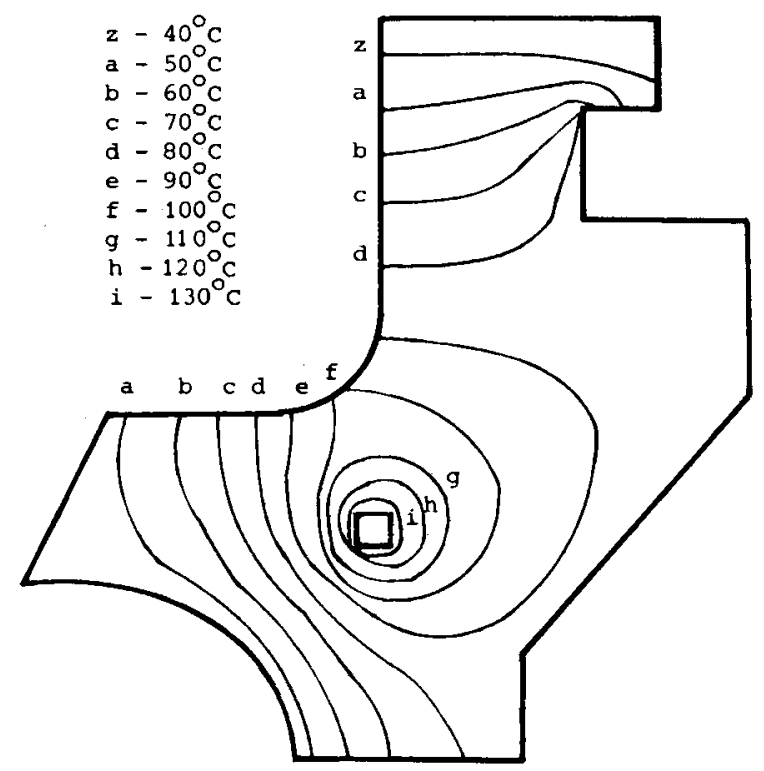

Figure 21. Pump casing-temperature field given by RIBEM

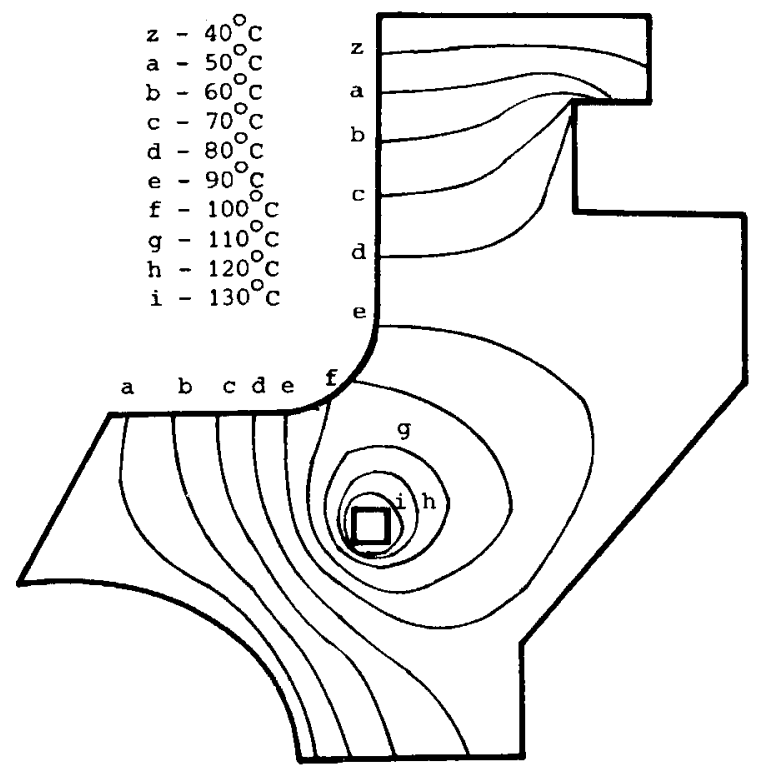

Figure 22. Pump casing-temperature field given by FEM

of Figure 20. In solving for the primary unknowns, FEM took $58 \mathrm{sec}$ while the Regular Indirect Method took a total time of only $16 \mathrm{sec}$ to invert the matrices and to solve for the interfacial temperatures. However, RIBEM required $117.3 \mathrm{sec}$ to set up the matrices compared to $65 \mathrm{sec}$ taken by the Finite Element Method. 
Table II. Comparison of computational effort for the pump casing problem

\begin{tabular}{|c|c|c|c|c|c|c|c|}
\hline \multirow{2}{*}{$\begin{array}{l}\text { Computing time } \\
\text { in: }\end{array}$} & \multirow[b]{2}{*}{1} & \multirow[b]{2}{*}{2} & \multirow[b]{2}{*}{3} & \multicolumn{2}{|c|}{$\begin{array}{c}\text { RIBEM } \\
\text { Subregions }\end{array}$} & \multirow[b]{2}{*}{ Total } & \multirow[b]{2}{*}{ FEM } \\
\hline & & & & 4 & 5 & & \\
\hline $\begin{array}{l}\text { Input Data } \\
\text { Processing }\end{array}$ & - & - & - & - & - & 1.0 & 50 \\
\hline $\begin{array}{l}\text { Setting up } \\
\text { Matrix }\end{array}$ & $16 \cdot 1$ & 11.9 & $20 \cdot 9$ & $23 \cdot 6$ & $7 \cdot 43$ & 79.93 & 65 \\
\hline $\begin{array}{l}\text { Inversion/ } \\
\text { Reduction }\end{array}$ & $2 \cdot 84$ & 1.88 & 4.07 & $4 \cdot 81$ & 0.96 & 14.56 & 58 \\
\hline $\begin{array}{l}\text { Interface } \\
\text { Matrix: } \\
\text { Setting up } \\
\text { Solving }\end{array}$ & - & - & - & - & - & $\begin{array}{c}37.4 \\
1.58\end{array}$ & - \\
\hline $\begin{array}{l}\text { Interior } \\
\text { Solution }\end{array}$ & - & - & - & - & - & 0.26 & - \\
\hline TOTAL & & & & & & 133.73 & 173 \\
\hline
\end{tabular}

All times are measured in sec on ICL 1906S system

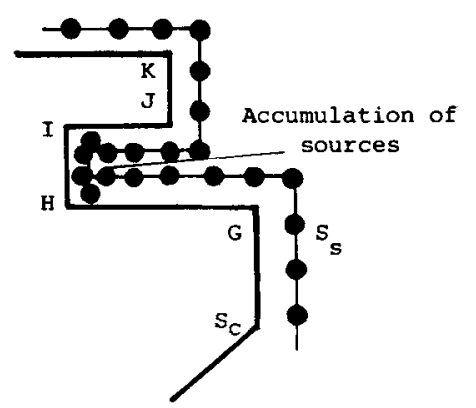

Figure 23. Section GHIJK of pump casing

The dimensions of the system matrix, when a conventional subregioning technique is employed in the Indirect Method, are given by

$$
\left[\sum_{i=1}^{n} N_{i}\right]^{2}
$$

where $N_{i}$ is the total number of degrees of freedom for subregion ' $i$ ' and $n$ is the number of subregions. For the pump casing problem this would give a $(293 \times 293)$ matrix. But in the present formulation the maximum size of a matrix to be inverted was $(72 \times 72)$. Hence, the ability of the conventional method to compete with the present one depends almost entirely on the kind of reduction scheme available to take advantage of the block banded matrix of the former.

In the Regular Method, subregioning avoids the problem of accumulation of source nodes in concave sections of the problem geometry. Take the case of section GHIJK of the boundary, as 


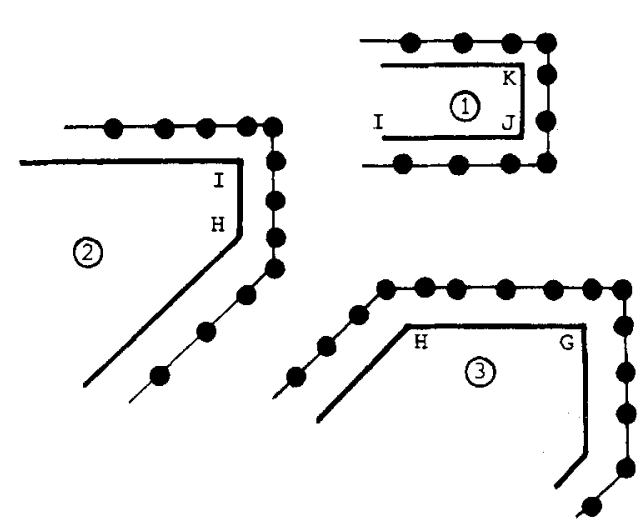

Figure 24. Subregioning of pump casing in RIBEM to avoid accumulation of sources in section GHIJK

shown in Figure 23. If the structure had been modelled without using subregions, the simple mechanism of shifting the source surface along the outward normal to the boundary would have resulted in the accumulation of sources in this section. In this situation, an easy solution was to subregion the section, as shown in Figure 24.

\section{CONCLUSIONS}

The Indirect BEM is computationally advantageous over its Direct Counterpart on two counts. Firstly, for the generation of internal solution, it requires the intergral of a single quantity $\phi$ (source density) as against the Direct BEM which involves the evaluation of the boundary integrals of both the field variables (temperatures and fluxes).

Direct BEM:

$$
u_{\mathrm{c}}=\int_{S} w q \mathrm{~d} S-\int_{S} f u \mathrm{~d} S
$$

Indirect BEM:

$$
u_{\mathrm{c}}=\int_{S} w \phi \mathrm{d} S
$$

Moreover, the IBEM requires only first order derivatives to calculate the internal fluxes, whereas the DBEM requires the second order derivatives of the fundamental solution $w$. Secondly, in the IBEM, temperatures and fluxes are indirectly linked by the distribution of the source density $\phi$ :

$$
\begin{aligned}
& u_{\mathrm{c}}=\int_{S} w \phi \mathrm{d} S \\
& q_{\mathrm{c}}=\int_{S} f \phi \mathrm{d} S
\end{aligned}
$$

where $f=\partial w / \partial n$. This natural feature of the method gives the opportunity to deal with each subregion of a multidomain problem separately. A new subregioning scheme has been formulated which eliminates the need for a sophisticated reduction scheme, implying reduction in computing overhead and saving in inversion time.

In the conventional (singular) form of the IBEM the source density $\phi$, which appears in the integral equations of the method, is distributed on the actual physical boundary of the problem. This approach has two distinct disadvantages. Firstly, it requires the accurate evaluation of certain singular integrals, which adds to the computational overhead. Secondly, and more 
importantly, in problems with geometric discontinuities, the source density $\phi$ becomes infinite at some of these locations. This gives rise to difficulties in modelling these regions, subsequently leading to errors. A regular variant of the IBEM has, therefore, been adopted whereby the surface of the source distribution is taken outside the physical domain of the problem. This approach overcomes the difficulties faced by the Singular IBEM and gives solutions the quality of which is maintained right up to the geometrical corners.

ACKNOWLEDGEMENT

The work presented in this paper is supported by the U.K. Science \& Engineering Research Council.

\section{REFERENCES}

1. C. A. Brebbia, The Boundary Element Method for Engineers, Pentech Press, London, 1978.

2. P. K. Banerjee and R. Butterfield, Boundary Element Methods in Engineering Science, McGraw-Hill, London, 1981.

3. V. D. Kupradze, Potential Methods in the Theory of Elasticity, Israel Program for Scientific Translations, Jerusalem, 1965.

4. J. L. Wearing, C. Patterson, M. A. Sheikh and A. G. Abul Rahman, 'A regular indirect boundary element method for heat conduction', Numerical Methods in Thermal Problems, Pineridge Press, Swansea, 1987.

5. A. G. Abdul Rahman, 'An investigation of the regular indirect boundary element method', Ph.D. Thesis, University of Sheffield, 1985 .

6. C. A. Brebbia and S. Walker, Boundary Element Techniques in Engineering, Newnes-Butterworths, London, 1980.

7. C. Patterson, J. L. Wearing and M. A. Sheikh, 'A review of recent developments in boundary domain techniques', Boundary Element Methods-Theory and Applications, The Institute of Physics, London, 1986.

8. J. L. Wearing, C. Patterson, M. A. Sheikh and A. G. Abdul Rahman, 'On the application of the regular indirect boundary element method for axisymmetric thermal analysis using subregions', Boundary Elements $I X$, SpringerVerlag, Berlin, 1987.

9. C. Patterson and M. A. Sheikh, 'Interelement continuity in the boundary element method', Topics in Boundary Element Research, Vol. I, Springer-Verlag, Berlin, 1984

10. M. Abramowitz and I. A. Stegun, Handbook of Mathematical Functions, Dover, New York, 1974.

11. V. S. Aypaci, Conduction Heat Transfer, Addison-Welsey, Reading, MA, U.S.A., 1966. 